\title{
Supersonic jets of hydrogen and helium for laser wakefield acceleration
}

\author{
K. Svensson, ${ }^{*}$ M. Hansson, F. Wojda, L. Senje, M. Burza, B. Aurand, G. Genoud, \\ A. Persson, C.-G. Wahlström, and O. Lundh ${ }^{\dagger}$ \\ Department of Physics, Lund University, P.O. Box 118, SE-221 00 Lund, Sweden
}

(Received 1 December 2015; published 2 May 2016)

\begin{abstract}
The properties of laser wakefield accelerated electrons in supersonic gas flows of hydrogen and helium are investigated. At identical backing pressure, we find that electron beams emerging from helium show large variations in their spectral and spatial distributions, whereas electron beams accelerated in hydrogen plasmas show a higher degree of reproducibility. In an experimental investigation of the relation between neutral gas density and backing pressure, it is found that the resulting number density for helium is $~ 30 \%$ higher than for hydrogen at the same backing pressure. The observed differences in electron beam properties between the two gases can thus be explained by differences in plasma electron density. This interpretation is verified by repeating the laser wakefield acceleration experiment using similar plasma electron densities for the two gases, which then yielded electron beams with similar properties.
\end{abstract}

DOI: 10.1103/PhysRevAccelBeams.19.051301

The development of bright and ultrashort sources of particles and $\mathrm{x}$ rays is an important area of research. Such sources are of interest in many domains, including materials science, chemistry, biology, and medicine. Currently, emerging sources based on laser-plasma acceleration [1] are attracting significant attention. The accelerator can be very compact, and the particle beams have several unique characteristics. Recent achievements include the generation of electron beams with high energies (few GeV) [2], short pulse duration (few femtoseconds) [3], high peak current (few kA) [4], low energy spread $(<1.5 \%)$ [5], and low emittance (few $\mathrm{mm} \times \mathrm{mrad}$ ) [6]. For most demanding applications, however, the stability of the source is also very important. A critical issue for laser wakefield accelerator (LWFA) research is to find ways to decrease shot-toshot fluctuations.

In a typical LWFA, an intense laser pulse is focused in a neutral gas medium and atoms, or molecules, are rapidly ionized by the leading edge of the laser pulse. The main part of the pulse interacts with a plasma, and free electrons are displaced by the laser ponderomotive force which leads to a significant charge separation and a copropagating plasma wave. Strong accelerating electric fields $(\sim 100 \mathrm{GV} / \mathrm{m})$ are present in the plasma wave, and copropagating electrons can be accelerated to high energies if they have sufficient initial kinetic energy and are located in an appropriate phase of the plasma wave. In the so-called bubble regime [7], the injection of electrons can be

*kristoffer.svensson@fysik.lth.se

†olle.lundh@fysik.lth.se

Published by the American Physical Society under the terms of the Creative Commons Attribution 3.0 License. Further distribution of this work must maintain attribution to the author(s) and the published article's title, journal citation, and DOI. achieved by driving the plasma wave to such a high amplitude that the wave breaks. This occurs as the velocity of the electrons exceeds the phase velocity of the plasma wave and results in self-injection of electrons from the background plasma into the accelerating phase of the plasma wave.

The threshold for wave breaking can be described as a laser power threshold [8] as well as a laser energy threshold [9] for a given plasma electron density $n_{e}$. Thus, for a given set of laser parameters, the self-injection threshold can be found by adjusting $n_{e}$. Assuming ideal gas behavior, the neutral gas number density $n$ in a supersonic jet is proportional to the pressure $p_{0}$ supplied to the nozzle and for a fully ionized gas $n_{e}=N_{e} n$, where $N_{e}$ is the number of electrons per atom, or molecule, depending on the gas species. Thus, for fully ionized gases, $n_{e} \propto p_{0}$. In this article, we present, to our knowledge, the first comparative study of electron beams emerging from supersonic jets of $\mathrm{H}_{2}$ and He. These gases were chosen since they will be fully ionized for the present experimental conditions.

The experimental investigations were conducted using the multiterawatt laser at the Lund Laser Centre. This Ti: sapphire-based system produced $37 \mathrm{fs}$ duration laser pulses with $650 \mathrm{~mJ}$ of energy on target during the present study. An $f / 15$ parabolic mirror focused the laser pulse to a $16 \mu \mathrm{m}$ (FWHM) spot measured in vacuum, which yielded a peak intensity of $5.7 \times 10^{18} \mathrm{~W} / \mathrm{cm}^{2}$. The beam waist was positioned, within one Rayleigh length, at the front edge of a supersonic gas flow released from a $2 \mathrm{~mm}$ diameter nozzle. Behind the interaction medium, along the laser propagation axis, a permanent dipole magnet dispersed the accelerated electrons according to energy. The dispersed electron beams impacted on a scintillating screen, imaged using a 16-bit digital camera. The integrated charge above 
the spectrometer threshold energy $(40 \mathrm{MeV})$ was also estimated using the measured response of the scintillator screen $[10,11]$.

In Fig. 1, two five-image sequences of electron beams accelerated in $2 \mathrm{~mm}$ gas jets of $\mathrm{H}_{2}$ and $\mathrm{He}$ operated at $p_{0}=9.5 \mathrm{bar}$ are presented. It is apparent that electron beams originating from $\mathrm{H}_{2}$ [Fig. 1(a)] were, compared to those accelerated in He plasmas [Fig. 1(b)], more stable in terms of maximum electron energy, position, and spatial divergence, as well as integrated beam charge. Most electron energy spectra contained a single peak with a relatively large energy spread, corresponding to the dispersed electron beams shown in Fig. 1(a). Also, the individual images shown in Fig. 1(a) are similar to the average of the full sequence, consisting of ten images, which is shown in Fig. 1(c). However, the electron beams emerging from He [Fig. 1(b)] fluctuated significantly and suffered from filamentation, which was not the case for (a)

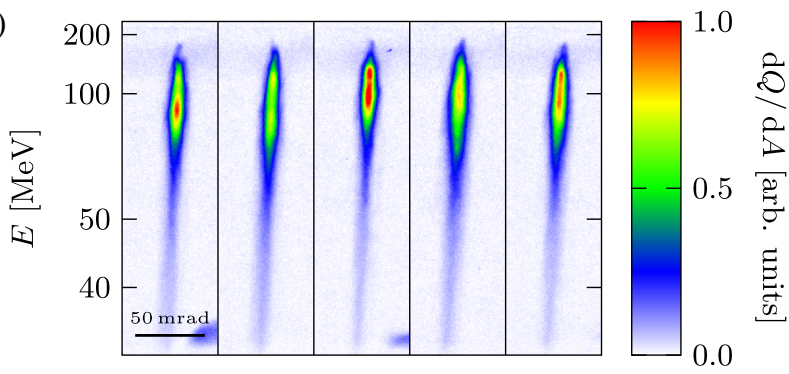

(b)

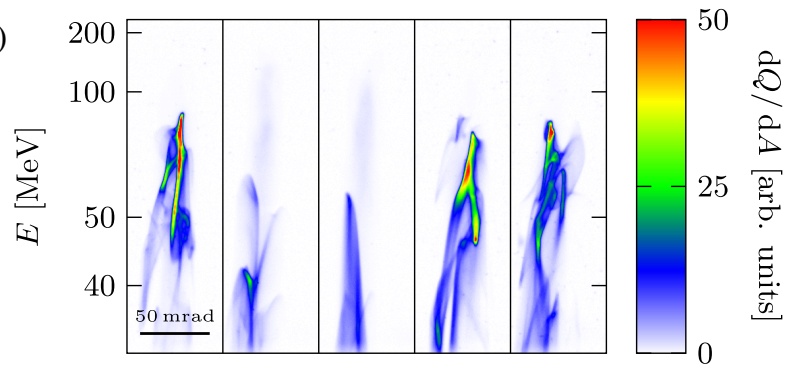

(c)

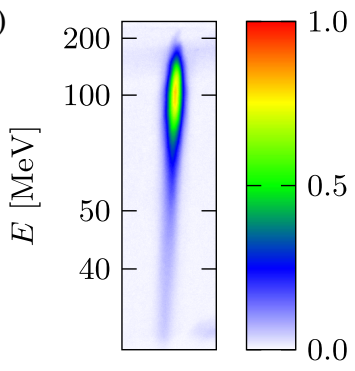

(d)

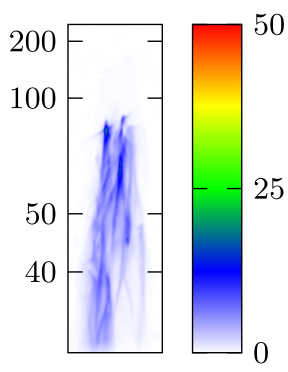

50

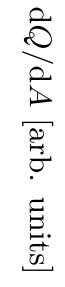

FIG. 1. False-color images of five electron beams emerging from (a) $\mathrm{H}_{2}$ and (b) He dispersed by a permanent dipole magnet. In both cases, a $2 \mathrm{~mm}$ nozzle was used at a fixed backing pressure of 9.5 bar. The reproducibility of the data is shown by the average of ten individual images of electron spectra for beams emerging from (c) $\mathrm{H}_{2}$ and (d) He. All color scales are normalized to the maximum signal in (a). beams from $\mathrm{H}_{2}$. Most of the energy spectra of the beams originating in $\mathrm{He}$ had multiple peaks, each often having very small energy spreads. It is also apparent that the sequence average [see Fig. 1(d)] is not similar to any of the individual images shown in Fig. 1(b). When comparing the two series, it can also be deduced that the integrated charge of beams accelerated in $\mathrm{He}$ is significantly larger than those accelerated in $\mathrm{H}_{2}$.

The integrated beam charge was measured in a sequence of pulses while varying the pressure in the range 3-15 bar, and the results are shown in Fig. 2. As can be seen, the threshold for self-injection, which is the point where beam charge increases rapidly, is at 9 bar for He but occurs at 11 bar for $\mathrm{H}_{2}$, indicating differences between the two media.

We have evaluated several phenomena in order to explain our observations, such as differences in the neutral gas ionization and the corresponding ionization-induced defocusing [12]. However, the intensity needed [13] for $\mathrm{He} \rightarrow \mathrm{He}^{+}$is $1.4 \times 10^{15} \mathrm{~W} / \mathrm{cm}^{2}$, and for $\mathrm{He}^{+} \rightarrow \mathrm{He}^{2+}$ is $8.8 \times 10^{15} \mathrm{~W} / \mathrm{cm}^{2}$, which are at least 2 orders of magnitude below the peak laser intensity used in this experiment. Thus, this effect should have been noticeable only at the front of the laser pulse and in the wings. Simulations of the laser-pulse evolution performed using the code WAKE [14], which included ionization of neutral gases, did not show any significant differences in pulse characteristics when propagating through $\mathrm{H}_{2}$ compared to $\mathrm{He}$ at identical $n_{e}$.

Another possible cause for the behavior in Fig. 2 could be weaker accelerating fields for $\mathrm{H}_{2}$ than for He. Since $\mathrm{H}_{2}$ is a molecular gas, the background of positively charged ions in the bubble behind the laser pulse might not be homogenous, as is expected for monatomic gases such as He. Assuming that the protons of the fully ionized $\mathrm{H}_{2}$ ions

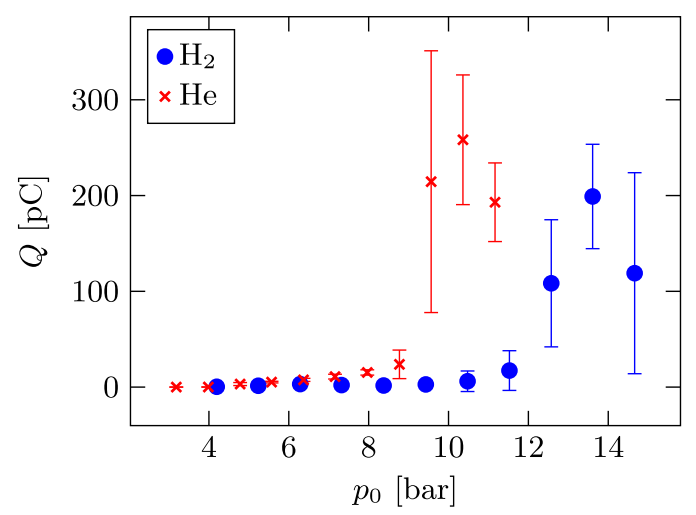

FIG. 2. Measured charge $Q$ in the electron beams accelerated in a $2 \mathrm{~mm}$ gas jet over the scanned pressure range 3-15 bar in $\mathrm{H}_{2}$ (blue circles) and $\mathrm{He}$ (red crosses) plotted as functions of the backing pressure. Each point represents the average of ten individual measurements with error bars indicating one standard deviation in each direction. Note that only electrons with an energy exceeding the cutoff ( $40 \mathrm{MeV}$ ) contributes to $Q$ in this figure. 
are separated by their molecular bond distance $(0.074 \mathrm{~nm})$, Coulomb repulsion will cause an explosion. However, in a $\mathrm{H}_{2}$ plasma, the initial ion speed $(\sim 4.5 \mathrm{~nm} / \mathrm{fs})$ is too small to have a noticeable effect on the ion density in the bubble.

Finally, the differences between the gases seen in Figs. 1 and 2 can be due to fluid mechanical differences between the gases. To determine the magnitude of such an influence on the resulting $n_{e}$, a simple model of a convergingdiverging nozzle was investigated. The relation between the nozzle throat sizes and flow Mach number M is [15]

$$
\left(\frac{r_{0}}{r^{*}}\right)^{2}=\frac{1}{\mathrm{M}}\left[\frac{2+(\kappa-1) \mathrm{M}^{2}}{(\kappa+1)}\right]^{\frac{\kappa+1}{2(\kappa-1)}}
$$

where $r_{0}$ is the nozzle exit radius, $r^{*}$ is the critical radius where the flow reaches sonic speeds inside the nozzle, and $\kappa$ is the ratio of specific heats of the gas with numerical values 1.41 for $\mathrm{H}_{2}$ and 1.66 for $\mathrm{He}$ [16]. For the specified $r^{*}=0.39 \mathrm{~mm}$ of the $2.0 \mathrm{~mm}$ diameter nozzle used in the experiments, Eq. (1) yields $\mathrm{M}=3.5$ and $\mathrm{M}=4.2$, for $\mathrm{H}_{2}$ and $\mathrm{He}$, respectively. Assuming that the gas can be described as an ideal gas, it is also possible to express the density at the nozzle exit, $n_{\text {exit }}$, as [17]

$$
n_{\text {exit }}=\frac{p_{0}}{k_{B} T_{0}}\left[1+\frac{\kappa-1}{2} \mathrm{M}^{2}\right]^{-\frac{1}{\kappa-1}},
$$

where $k_{B}$ is Boltzmann's constant and $T_{0}=293 \mathrm{~K}$ the temperature. As the flow exits the nozzle, it will diverge with half-angle $\varphi$ given by $\varphi=\alpha+\theta$, where $\alpha=$ $\arcsin \mathrm{M}^{-1}$ is the Mach cone half-angle and $\theta$ the nozzle expansion angle. This means that, using cylindrical symmetry, the radius of the gas flow can be written as $r=r_{0}+h \tan \varphi$, where $h$ is the vertical distance from the nozzle exit. Assuming that $\varphi$ remains constant, the gas density at a specific $h$ close to the nozzle exit can be estimated as $n=n_{\text {exit }}\left(r_{0} / r\right)^{2}$.

As is seen from Eqs. (1) and (2), there is a nontrivial relation between $n_{\text {exit }}$ and the gas-species-dependent $\kappa$. Therefore, characterizing the relation between $p_{0}$ and $n$ for both gases released from the nozzle was necessary and performed experimentally. The phase shift introduced by He at $n=5 \times 10^{18} \mathrm{~cm}^{-3}$ over $2 \mathrm{~mm}$ for $633 \mathrm{~nm}$ light is $0.14 \mathrm{rad}$ (corresponding to a $14 \mathrm{~nm}$ optical path length difference), which is difficult to measure with an ordinary interferometer. Therefore, $n$ was measured as a function of $p_{0}$ with a setup consisting of an expanded HeNe-laser beam and a wave-front sensor [18], which is sensitive enough to determine the phase shift introduced by He. By assuming full ionization, $n_{e}$ is then plotted as a function of $p_{0}$ for the two gases in Fig. 3, which clearly shows that they resulted in different $n_{e}$ at all $p_{0}$. Using $r^{*}$ as a fitting parameter in Eq. (1) to simultaneously fit the theoretical model to experimental results obtained for both $\mathrm{H}_{2}$ and He yielded $r^{*} \approx 0.35 \mathrm{~mm}$, which is close to the specified critical radius

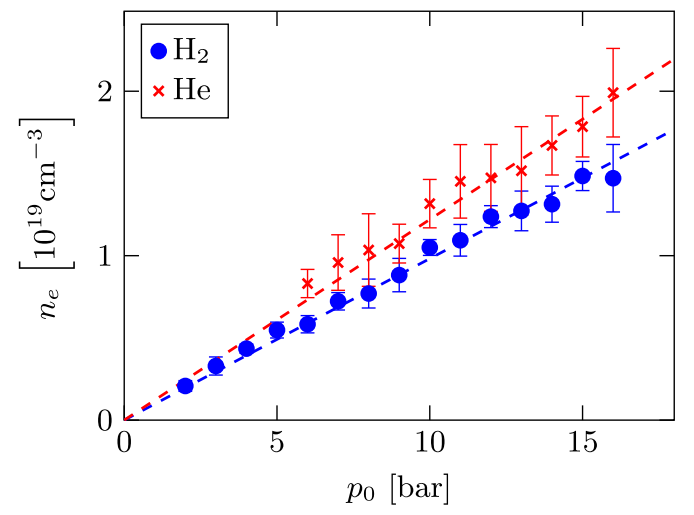

FIG. 3. The plasma electron number density $n_{e}, 1 \mathrm{~mm}$ from the nozzle orifice ( $2 \mathrm{~mm}$ diameter) as a function of the applied backing pressure $\left(p_{0}\right)$ for $\mathrm{H}_{2}$ (blue circles) and He (red crosses). Assuming full ionization, the plateau electron number density $n_{e}$ along the center axis in the laser propagation direction is determined from measurements of the neutral gas number density (n) using a setup consisting of an expanded HeNe beam and a wave-front sensor. Each point represents the average of 10-20 individual measurements, and the error bars indicate one standard deviation in each direction. The dashed lines are the theoretical results fitted with regards to $r^{*}$.

of the nozzle. The fitted results, shown as dashed lines in Fig. 3, are in excellent agreement with the experimental data.

From the theoretical model, it was found that $n_{\mathrm{He}} \approx 1.3 n_{\mathrm{H}_{2}}$. Thus, $n_{e}$ in $\mathrm{He}$ is $\sim 30 \%$ higher than for $\mathrm{H}_{2}$ at any specific $p_{0}$. Compensating for this difference and plotting the data in Fig. 2 as a function of $n_{e}$ instead of $p_{0}$ results in Fig. 4(a). Now it can be seen that the rapid increase in $Q$ occurs at the same $n_{e}$ for both gas species. The effect observed in Fig. 1 is therefore not significantly due to any of the previously discussed differences between the two gas species but can be explained by the relation between $n_{e}$ and $p_{0}$ in Fig. 3. In Fig. 1, the electrons were accelerated in gas jets with $p_{0}=9.5$ bar which corresponds to $n_{e}=9.3 \times 10^{18} \mathrm{~cm}^{-3}$ for $\mathrm{H}_{2}$ and $n_{e}=1.2 \times 10^{19} \mathrm{~cm}^{-3}$ for He. Using a similar $n_{e}$ for $\mathrm{He}$ as for $\mathrm{H}_{2}$ in Fig. 1(a) results in Fig. 4(b). Now, the accelerated electron beams emerging from $\mathrm{He}$ are very similar to the ones from $\mathrm{H}_{2}$, which is also seen when comparing the averages of ten individual images in Figs. 1(c) $\left(\mathrm{H}_{2}\right)$ and 4(c) (He). Laser self-focusing inside the plasma becomes stronger with increasing $n_{e}$, resulting in a smaller spot size $w_{0}$ and a higher normalized vector potential $a_{0}$ for $\mathrm{He}$ than for $\mathrm{H}_{2}$. For small $w_{0}$ and high $a_{0}$, it is expected that self-injection LWFA results in unstable, high charge electron beams, since transversal injection dominates over longitudinal injection [19]. When longitudinal injection is the dominant injection mechanism (large $w_{0}$ and small $a_{0}$ ), the accelerated electron beams becomes very stable, but with low charge. Thus, the differences seen in Fig. 1 can be explained by the differences in $n_{e}$ between $\mathrm{H}_{2}$ and $\mathrm{He}$ at 

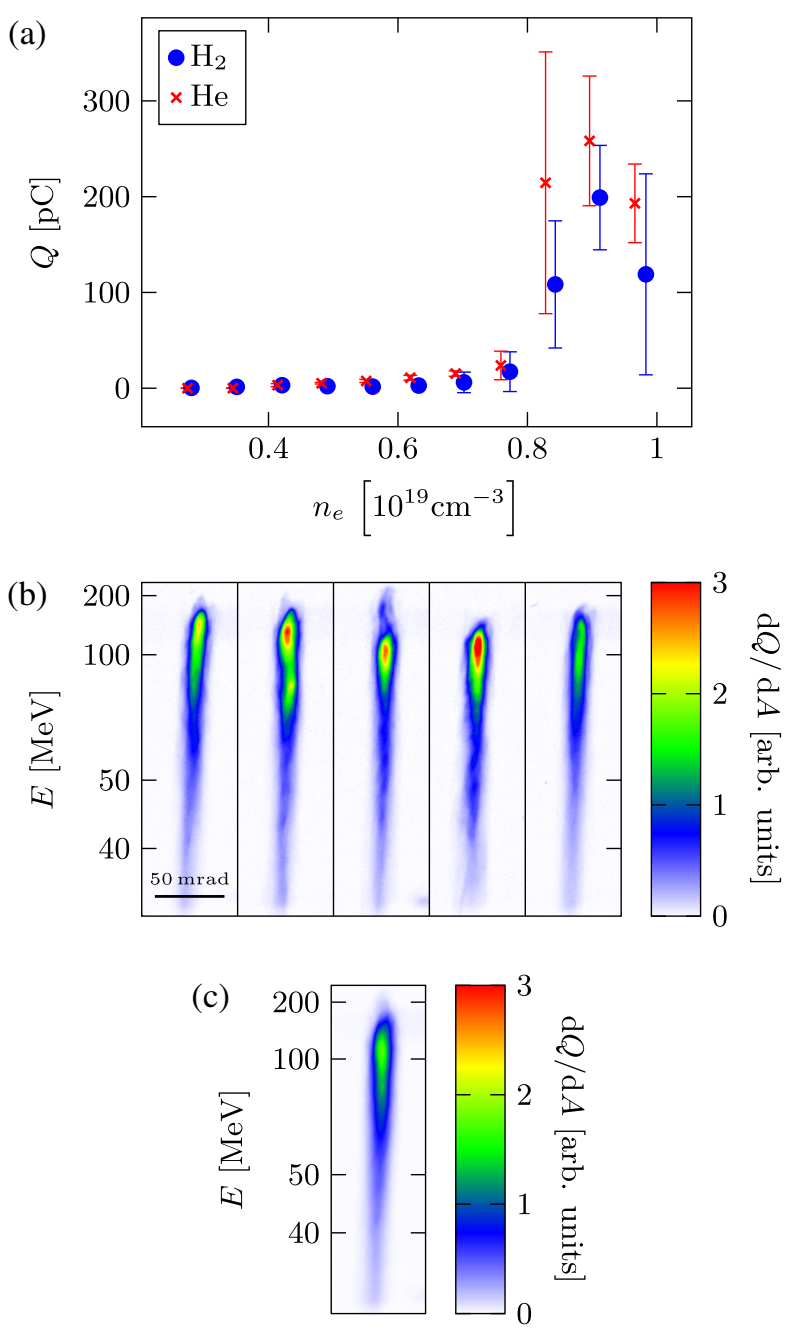

FIG. 4. (a) Measured charge $Q$ from Fig. 2 plotted against plasma electron density $n_{e}$ (assuming full ionization) instead of backing pressure $p_{0}$, by using the results of Fig. 3. Again, only electrons with energies exceeding the cutoff energy of the spectrometer setup contribute to $Q$. Each point represents the average of ten individual measurements with error bars indicating one standard deviation. (b) False-color images of five electron beams emerging from $\mathrm{He}$ at $n_{e}=9.8 \times 10^{18} \mathrm{~cm}^{-3}$ dispersed by a permanent dipole magnet, showing a high resemblance to electron beams accelerated in $\mathrm{H}_{2}$ at similar $n_{e}$. (c) Average of the full ten-image sequence which is partly shown in (b). The color scales are normalized to the maximum signal in Fig. 1(a).

identical $p_{0}$, since the two series have different injection mechanisms.

In this study, we have shown that electron beams emerging from $\mathrm{H}_{2}$ and $\mathrm{He}$ at identical nozzle backing pressures have different properties. This is found to be primarily a result of the supersonic gas jet number density dependence on a specific heat ratio which, generally, differs between gas species. Repeating the experiment using similar $n_{e}$ for both gases confirms these findings, since the resulting beams of accelerated electrons then showed similar properties regardless of gas species. Thus, both gases resulted in stable, low charge electron beams for $n_{e}<8 \times 10^{18} \mathrm{~cm}^{-3}$, which can be deduced from Fig. 4 . It is also believed that this effect can have implications when using gas mixtures as an acceleration medium and should be studied further.

We acknowledge the support of the Swedish Research Council, the Knut and Alice Wallenberg Foundation, the Swedish Foundation for Strategic Research, Laserlab-Europe/CHARPAC (Grant Agreement No. 284464, EC's 7th Framework Programme) and EuCARD2/ANAC2 (Grant Agreement No. 312453, EC's 7th Framework Programme).

[1] T. Tajima and J. M. Dawson, Laser Electron Accelerator, Phys. Rev. Lett. 43, 267 (1979).

[2] W. P. Leemans, A. J. Gonsalves, H. S. Mao, K. Nakamura, C. Benedetti, C. B. Schroeder, C. Toth, J. Daniels, D. E. Mittelberger, S. S. Bulanov, J. L. Vay, C. G. R. Geddes, and E. Esarey, Multi-GeV Electron Beams from CapillaryDischarge-Guided Subpetawatt Laser Pulses in the SelfTrapping Regime, Phys. Rev. Lett. 113, 245002 (2014).

[3] A. Buck, M. Nicolai, K. Schmid, C. M. S. Sears, A. Sävert, J. M. Mikhailova, F. Krausz, M. C. Kaluza, and L. Veisz, Real-time observation of laser-driven electron acceleration, Nat. Phys. 7, 543 (2011).

[4] O. Lundh, J. Lim, C. Rechatin, L. Ammoura, A. Ben-Ismail, X. Davoine, G. Gallot, J.-P. Goddet, E. Lefebvre, V. Malka, and J. Faure, Few femtosecond, few kiloampere electron bunch produced by a laser-plasma accelerator, Nat. Phys. 7, 219 (2011).

[5] C. Rechatin, J. Faure, A. Ben-Ismaïl, J. Lim, R. Fitour, A. Specka, H. Videau, A. Tafzi, F. Burgy, and V. Malka, Controlling the Phase-Space Volume of Injected Electrons in a Laser-Plasma Accelerator, Phys. Rev. Lett. 102, 164801 (2009).

[6] R. Weingartner, S. Raith, A. Popp, S. Chou, J. Wenz, K. Khrennikov, M. Heigoldt, A. R. Maier, N. Kajumba, M. Fuchs, B. Zeitler, F. Krausz, S. Karsch, and F. Grüner, Ultralow emittance electron beams from a laser-wakefield accelerator, Phys. Rev. Accel. Beams 15, 111302 (2012).

[7] A. Pukhov and J. Meyer-ter-Vehn, Laser wake field acceleration: The highly non-linear broken-wave regime, Appl. Phys. B 74, 355 (2002).

[8] D. H. Froula, C. E. Clayton, T. Döppner, K. A. Marsh, C. P. J. Barty, L. Divol, R. A. Fonseca, S. H. Glenzer, C. Joshi, W. Lu, S. F. Martins, P. Michel, W. B. Mori, J. P. Palastro, B. B. Pollock, A. Pak, J. E. Ralph, J. S. Ross, C. W. Siders, L. O. Silva, and T. Wang, Measurements of the Critical Power for Self-Injection of Electrons in a Laser Wakefield Accelerator, Phys. Rev. Lett. 103, 215006 (2009).

[9] S. P. D. Mangles, G. Genoud, M. S. Bloom, M. Burza, Z. Najmudin, A. Persson, K. Svensson, A. G. R. Thomas, and C.-G. Wahlström, Self-injection threshold in self-guided laser wakefield accelerators, Phys. Rev. Accel. Beams 15, 011302 (2012). 
[10] Y. Glinec, J. Faure, A. Guemnie-Tafo, V. Malka, H. Monard, J. P. Larbre, V. De Waele, J. L. Marignier, and M. Mostafavi, Absolute calibration for a broad range single shot electron spectrometer, Rev. Sci. Instrum. 77, 103301 (2006).

[11] A. Buck, K. Zeil, A. Popp, K. Schmid, A. Jochmann, S. D. Kraft, B. Hidding, T. Kudyakov, C. M. S. Sears, L. Veisz, S. Karsch, J. Pawelke, R. Sauerbrey, T. Cowan, F. Krausz, and U. Schramm, Absolute charge calibration of scintillating screens for relativistic electron detection, Rev. Sci. Instrum. 81, 033301 (2010).

[12] T. Auguste, P. Monot, L.-A. Lompré, G. Mainfray, and C. Manus, Defocusing effects of a picosecond terawatt laser pulse in an underdense plasma, Opt. Commun. 89, 145 (1992).

[13] P. Gibbon, Short Pulse Laser Interactions with Matter, An Introduction (Imperial College Press, London, 2005).

[14] P. Mora and J. Thomas M. Antonsen, Kinetic modeling of intense, short laser pulses propagating in tenuous plasmas, Phys. Plasmas 4, 217 (1997).
[15] S. Semushin and V. Malka, High density gas jet nozzle design for laser target production, Rev. Sci. Instrum. 72, 2961 (2001).

[16] C. Nordling and J. Österman, Physics Handbook, 7th ed. (Studentlitteratur, Lund, 2004).

[17] K. Schmid and L. Veisz, Supersonic gas jets for laser-plasma experiments, Rev. Sci. Instrum. 83, 053304 (2012).

[18] G. R. Plateau, N. H. Matlis, C. G. R. Geddes, A. J. Gonsalves, S. Shiraishi, C. Lin, R. A. van Mourik, and W. P. Leemans, Wavefront-sensor-based electron density measurements for laser-plasma accelerators, Rev. Sci. Instrum. 81, 033108 (2010).

[19] S. Corde, C. Thaury, A. Lifschitz, G. Lambert, K. Ta Phuoc, X. Davoine, R. Lehe, D. Douillet, A. Rousse, and V. Malka, Observation of longitudinal and transverse selfinjections in laser-plasma accelerators, Nat. Commun. 4, 1501 (2013). 